\title{
Aplicación de conocimientos docentes en contenido curricular, pedagogía y tecnología a través de herramientas audiovisuales
}

\author{
Yánez Corrales, Angela Cristina; Moreano Barragan, Elsie Paulina \\ Aplicación de conocimientos docentes en contenido curricular, pedagogía y tecnología a través de herramientas \\ audiovisuales \\ Revista Educación, vol. 45, núm. 2, 2021 \\ Universidad de Costa Rica, Costa Rica \\ Disponible en: https://www.redalyc.org/articulo.oa?id=44066178023 \\ DOI: https://doi.org/10.15517/revedu.v45i1.43469
}

\section{(c) $(1) \Theta$}

Esta obra está bajo una Licencia Creative Commons Atribución-NoComercial-SinDerivar 3.0 Internacional. 


\title{
Aplicación de conocimientos docentes en contenido curricular, pedagogía y tecnología a través de herramientas audiovisuales
}

\author{
Use of Audiovisual Tools to Enhance Teaching of Curricular Content, Pedagogy and Technology
}

Angela Cristina Yánez Corrales

Pontificia Universidad Católica del Ecuador, Ecuador

acyanez.co@gmail.com

(iD https://orcid.org/0000-0002-2609-1858

Elsie Paulina Moreano Barragan

Pontificia Universidad Católica del Ecuador, Ecuador

epmoreano@puce.edu.ec

(iD) https://orcid.org/0000-0003-2027-5282
DOI: https://doi.org/10.15517/revedu.v45i1.43469

Redalyc: https://www.redalyc.org/articulo.oa? $\mathrm{id}=44066178023$

Recepción: 21 Agosto 2020

Aprobación: 25 Octubre 2020

\section{Resumen:}

Objetivo: Aplicar y evaluar herramientas audiovisuales para el aprendizaje con base en el modelo teórico TPACK (Technological Pedagogical and Content Knowledge): conocimiento curricular, conocimiento pedagógico y conocimiento de la tecnología. Método: Consiste en la aplicación del modelo de integración TPACK, donde se aplican las premisas Wicked, Creative. Package, utilizadas en herramientas y recursos tecnológicos para facilitar la docencia y la capacidad de adaptarse y renovarse en versiones y cambios educativos basados en la resolución creativa de problemas de aprendizaje. Para la evaluación se utilizaron técnicas cualitativas y cuantitativas. Resultados: Se realizaron videos educativos, los cuales consideraron las fases de preproducción, producción y de postproducción. La evaluación detalla de resultados con parámetros establecidos en: atención, curiosidad, utilidad en repaso, estímulo a aprender, ayuda al entendimiento y el deseo de reutilizarlos. Además, se realiza seguimiento del uso que han tenido estos videos en YouTube durante cuatro años; se exponen estadísticas de esta red social. Conclusiones: Utilizar conocimientos docentes en contenido, pedagogía y tecnología define una forma significativa y eficiente de enseñar con tecnología a través de videos educativos.

Palabras Clave: Tecnología de la educación, Formación del profesorado, Elaboración de medios de enseñanza, Tecnologías de la Información y la Comunicación, TIC, Teoría de la educación.

\section{Abstract:}

Objective: To use and assess audiovisual tools to enhance learning in the classroom, based on the theoretical TPACK (Technological Pedagogical Content Knowledge) model. Methodology: Application of the TPACK model which uses a" Wicked", "Creative" and "Package" approach, in which teachers are the designers of their educational tools which help them creatively resolve learning issues. Either qualitative or quantitative techniques were used for validation purposes. Results: Educational videos were produced involving a pre-production, production, and post-production phase. Results are based on parameters that include attention, curiosity, usefulness for review purposes, learning engagement, extent to which it helped students better understand the content and the desire to reuse these videos. Furthermore, since these videos are viewed on YouTube, the number of views has been tracked for the past four years. Conclusions: Integration of teacher knowledge with content, pedagogy and technology provides a meaningful and efficient way of teaching through the use of technology - specifically, educational videos.

KeYwords: Education technology, teacher training, Development of teaching aids, Information and Communication Technologies, ICT, Theory of education, Education technology, Teacher Training, Development of Teaching Aids, Information and Communication Technology, ICT, Educational Theory.. 


\section{INTRODUCCIÓN}

La docencia en la educación superior se caracteriza por tener un elevado grado de complejidad, debido a que la transferencia de conocimientos no solo aborda el aspecto didáctico o la forma de enseñar. Se enfatiza en la reflexión, el análisis y la interpretación de aspectos profesionales, con la interacción de diversos elementos, entre ellos lo social, institucional, entre otros (Fierro, Fortoul y Rosas, 2000; García, Loredo y Carranza, 2008).

Por su parte, las instituciones de educación superior exigen estilos de formación más integral y pertinente a través de modelos educativos específicos, para lo cual la articulación de las tecnologías de la información y la comunicación (TIC) es significativamente importante en la dinámica de desarrollo, específicamente en el proceso de aprendizaje (Morales, Trujillo y Raso, 2015).

En la carrera de enfermería de la Universidad Católica del Ecuador (PUCE), en el año 2013, se realiza el diagnóstico situacional sobre características básicas de las TIC: aplicación y conocimiento del aporte de la institución en TIC y el modelo educativo vigente en ella, con el fin de generar un análisis de fortalezas, debilidades, amenazas y oportunidades. En referencia al ámbito docente y al ámbito curricular, se encontró un déficit en el uso metodológico de los recursos o herramientas tecnológicas, así como la débil integración de contenido profesional tecnológico en el microcurrículo de enfermería (Yánez, Ortiz y Espinosa, 2016). En este contexto parte la pregunta de investigación: Cómo mejorar el proceso de enseñanza aprendizaje con el uso de las nuevas tecnologías en la asignatura de Anatomía y Fisiología de la carrera de Enfermería?

Este trabajo parte con el compromiso de mejorar el microcurrículo de la Carrera de Enfermería, y tiene como objetivo aplicar y validar herramientas educativas audiovisuales para el aprendizaje con base en el modelo teórico TPACK (Technological Pedagogical Content and Knowledge).

El modelo teórico de Koehler y Mishra: TPC Knowledge se enmarca en la adquisición de tres conocimientos por parte de la persona docente para llegar a la calidad. A) conocimiento de contenido de su materia o contenido curricular, B) conocimiento de pedagogía, en la que enfatiza el uso de estrategias, métodos, técnicas, actividades y herramientas de enseñanza para lograr incentivar el aprendizaje de contenidos y C) conocimiento de tecnología, que consiste en aplicar herramientas y recursos tecnológicos para facilitar la docencia y poseer capacidad de adaptarse y renovarse en versiones y cambios. La intersección de estos tres conocimientos define una forma significativa y eficiente de enseñar con tecnología, que supera el conocimiento aislado de los distintos elementos y son la base de la práctica educativa con TIC, ya que exige un fuerte elemento pedagógico y una comprensión de conceptos en el uso de determinadas tecnologías, las cuales aportan de forma constructiva en la enseñanza, con la característica de que pueden ayudar a compensar algunas de las dificultades de aprendizaje que el estudiantado enfrenta (Mishra y Koehler, 2006; Harris, Mishra y Koehler, 2009; Valverde, Garrido y Fernández, 2010).

En tanto, el fin de este trabajo es incorporar nuevas tecnologías del tipo audiovisual como una herramienta de aprendizaje accesible, práctica, novedosa y aplicable en todo tipo de enseñanza, sea presencial o virtual. De manera que permita cumplir con los resultados de aprendizaje y llegar a un perfil de egreso de una manera actualizada y entretenida para estudiantes, quienes son nativos y nativas digitales y consideran esta herramienta de aprendizaje más acorde con su realidad.

\section{Metodología}

\section{El desarrollo de esta investigación considera 2 fases:}

Fase 1 preproducción de herramientas educativas tecnológicas, donde se aplica el modelo de integración TPACK bajo las premisas Wicked, Creative y Package, las cuales permiten determinar cuál es el problema en cada asignatura de la malla curricular para la implementación de la tecnología, cuál es la solución más creativa y quién la desarrolla: 
- Teaching with technology is a WICKED (enseñar con tecnología es un problema complejo).

- Wicked problems require CREATIVE solutions (los problemas complejos requieren de soluciones creativas).

- Teachers are designers of the total PACKAGE (Los profesores son diseñadores del paquete completo).

Bajo la última premisa, el modelo considera 7 componentes integrados a partir de los 3 conocimientos (curriculares, pedagógicos y tecnológicos) que deben ser contemplados de forma individual y en su mutua interacción para el desarrollo de herramientas educativas (Valverde, et al., 2010):

- Conocimiento pedagógico (PK): Base de conocimiento sobre pedagogía, didáctica y métodos de enseñanza que debe poseer toda persona docente.

- Conocimiento disciplinar (CK): Trata del conocimiento que el personal docente debe poseer de la materia que va a impartir.

- Conocimiento tecnológico (TK): Todo lo relacionado con el conocimiento sobre las TIC.

- Conocimiento pedagógico disciplinar (PCK): El conocimiento de las didácticas de la materia. Integra el conocimiento de la disciplina y de cómo enseñarla. Afecta al conocimiento pedagógico y disciplinar. ¿Qué se va a enseñar?, ¿Cómo se va a enseñar?, ¿Qué actividades son las más adecuadas?, ¿Qué conocimientos previos se requieren?

- Conocimiento tecnológico disciplinar (TCK): Se trata del conocimiento sobre qué tecnologías son las más adecuadas para enseñar una materia concreta, pero, además, debe saber cuál es más adecuado para su utilización en la enseñanza concreta de un contenido.

- Conocimiento tecnológico pedagógico (TPK): Se trata del conjunto de saberes relacionado con el uso de las TIC en la educación. ¿Cómo se debe enseñar cuando se emplea tecnología?, ¿Qué situaciones son las más adecuadas?, aspectos positivos y negativos de su uso.

- Conocimiento tecnológico, pedagógico y disciplinar (TPACK): Es la integración de todos los componentes anteriores. Supone integrar lo que la persona docente sabe sobre la materia que desea impartir, los métodos didácticos más adecuados a la situación concreta del alumnado. Cómo integrar la tecnología para enseñar (mejor) un contenido concreto.

La Fase 2 considera la elaboración de la herramienta educativa audiovisual, la cual considera 3 etapas:

1. Preproducción para crear videos educativos: Se elige la temática, se crea el guion, se selecciona el escenario y se practica antes de la grabación.

2. Producción para crear videos educativos: Se realiza revisión y prueba de video, análisis del audio, duración, incorporación de los tres momentos del video.

3. Postproducción para crear videos educativos: Se realiza edición, publicación y evaluación del video.

En este contexto metodológico, se realizaron 7 videos de repaso de la asignatura Anatomía y Fisiología, en función de las necesidades de aprendizaje para procedimientos de enfermería y en consideración que las herramientas educativas audiovisuales (videos) utilizan diferentes canales perceptivos que facilitan el aprendizaje y el recuerdo. Se digitalizaron contenidos con base en un diseño instruccional. Por otra parte, la persona docente cumplió con el conocimiento curricular validado con el grado académico en correspondencia a la enseñanza de Anatomía y Fisiología. En cuanto al conocimiento pedagógico y tecnológico, la persona docente cuenta con una maestría en educación y tecnología, lo que permite el desarrollo adecuado de las herramientas (Ribayo, 2011).

Para cada video se escribió un guion, los cuales se analizaron junto con el personal docente de la asignatura, cuyo prerrequisito es Anatomía y Fisiología, contenidos significativos de la materia, necesarios para la 
realización de técnicas procedimentales de enfermería. Además, se contextualizaron en su utilidad para esta práctica profesional. El contenido de los videos se redactó con base en la experiencia docente y con ayuda de textos de Anatomía y Fisiología de los siguientes autores: Tórtora y Derrickson; Martini y Nath; Marieb y Hoehn, y textos digitales de la Carnegie Mellon University y de Openstax CNX College. Cada guión escrito tiene su correspondiente acompañamiento visual. Si son gráficos, estos provienen de la toma de fotografías de maquetas de la Facultad de Medicina de la PUCE, para ello se utilizó una cámara digital Samsung y se trabajaron por medio del programa Photos de Apple http://www.apple.com/osx/photos/. Provienen también de gráficos de uso abierto (Creative Commons) bajados de internet. Las fuentes de internet usadas para bajar gráficos son: Wikimedia Commons (https://commons.wikimedia.org/wiki/Mai n_Page), con gráficos de autores como Matthew Koehler, Patrick Lynch o Bruce Blausen entre otros, Open Learning Initiative of the Carnegie Mellon University (http://oli.cmu.edu/teach-with-oli/review-our-free -open-courses/), y OpenStax (https://openstaxcollege.org/textbooks/anatomy-and-physiology). Con estos gráficos se produjeron cortos animados por medio del programa de presentaciones de Apple llamado Keynote (http://www.apple.com/es/mac/keynote/). Se diseñó un formato con fondo negro y letras blancas para cada animación y se exportaron como grabación o grabación de pantalla por medio del programa Quicktime (ht tp://www.apple.com/es/quicktime/download/). Estos pequeños cortos de gráficos animados se montan en el programa iMovie (http://www.apple.com/es/ios/imovie/).

Seguidamente, con una filmadora se grabaron tomas cortas sobre contenidos, también se realizaron cortes de videos de uso abierto con el programa Quicktime, los cuales fueron subidos a iMovie.

En iMovie se armó cada video educacional definitivo en una línea del tiempo y se procedió a grabar la voz del guion por medio de un micrófono externo. Por último, se colocaron transiciones, títulos y créditos de cada corto utilizado y se exportó el video en archivo mp4 para ser subido a YouTube o a la plataforma Moodle de la PUCE.

\section{Evaluación de las herramientas educativas audiovisuales (videos)}

Para la evaluación se diseñó y aplicó una encuesta a docentes $(n=30)$ y estudiantes $(n=39)$ de la carrera de Enfermería de la PUCE, entre diciembre de 2014 y enero de 2015. La encuesta determina que estos videos pueden ser usados como: introducción a la materia, repaso al finalizar un capítulo, repaso previo a un examen o repaso en años futuros. Se solicita que se comparen los videos con otras formas utilizadas de repaso como son: repasar notas de clase, releer textos, revisar con compañeros, realizar cuestionarios o repasar presentaciones docentes, y se solicita que se califiquen los siguientes parámetros: atención, curiosidad, utilidad en repaso, estímulo a aprender, ayudar al entendimiento, y deseo de reutilización. La escala de calificación es de cuatro, donde la calificación de 4 indica que es superior, 3 es mejor, 2 es equivalente y 1 es inferior.

En la evaluación de los y las estudiantes (Figura 1), solicitaron repetir los videos observados para repasar la materia y rendir la evaluación parcial antes de responder la encuesta de evaluación.

En el caso del personal docente (Figura 2), este evaluó un video de su interés y procedió a llenar la encuesta. Del total de 30 docentes, 20 solicitaron ver más de un video antes de llenar la encuesta de evaluación

De todos los videos realizados, cada uno de los videos fue observado al menos en una ocasión. El material de la encuesta fue tabulado en Excel, donde se realizaron los cálculos y se graficaron los resultados.

Los videos fueron montados en la plataforma de YouTube; luego de cuatro años se realizó una revisión de sus estadísticas para validar el uso de estos videos en esta plataforma social.

\section{Resultados}

Al comparar otras formas de repaso de la asignatura de Anatomía y Fisiología con las herramientas educativas los audiovisuales elaboradas (videos), una calificación superior de $4 / 4$ fue conferida por el $82 \%(n=32)$ del 
estudiantado y el $83 \%(n=26)$ del profesorado encuestado, esto en cinco de los seis parámetros calificados: capturar atención, estimular a aprender, facilitar el repaso, ayudar a entender y deseo de reutilizarlos.

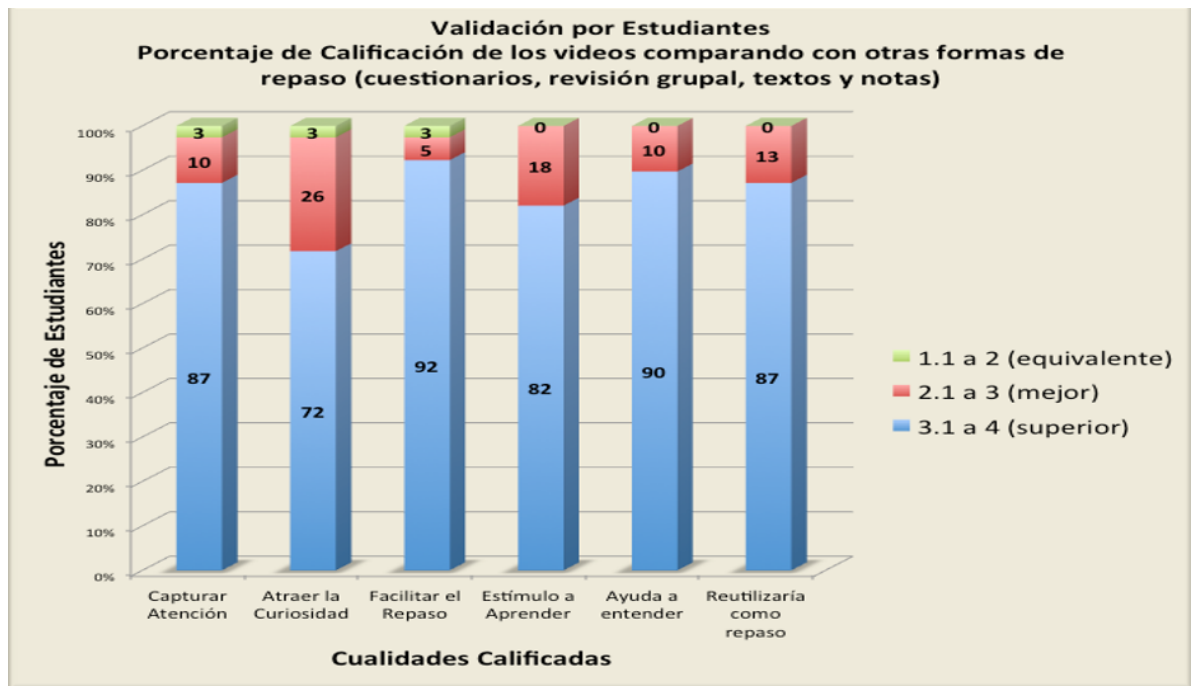

FIGURA 1.

Calificación dada por estudiantes de la carrera de Enfermería de la PUCE. al uso de los videos en comparación con otras formas de repaso (cuestionarios, revisiones grupales, textos y notas)

Fuente: Elaboración propia

Las respuestas de estudiantes a los parámetros Facilitar el repaso $92 \%(\mathrm{n}=36)$ y ayuda a atender $90 \%$ $(\mathrm{n}=95)$, reflejan el mayor número de respuestas versus el parámetro atraer la curiosidad $72 \%(\mathrm{n}=28)$, que demuestra el número de respuestas más bajo frente a la utilización de las herramientas educativas utilizadas (videos).

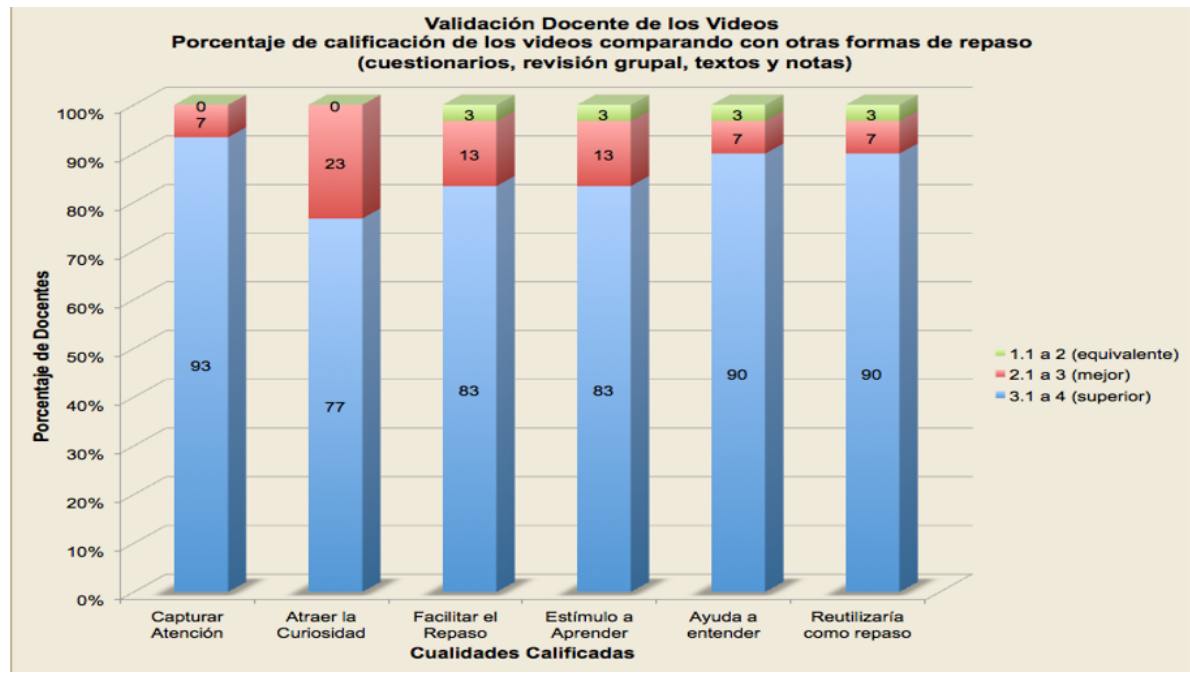

FIGURA 2.

Calificación dada por docentes de la Carrera de Enfermería de la PUCE a los videos en comparación con otras formas de repaso (cuestionarios, revisiones grupales, textos y notas)

Fuente: Elaboración propia

En relación con el personal docente, las respuestas a los parámetros Capturar la atención $93 \%(\mathrm{n}=28)$ refleja el mayor número de respuestas, caso contrario es el parámetro atraer la curiosidad $77 \%(\mathrm{n}=23)$, parámetro que coindice con la percepción del estudiantado. 
Las respuestas de estudiantes y docentes a los parámetros de estímulo a aprender (Figura 1 y Figura 2) y ayudar a entender (Figura 1 y Figura 2) coinciden. En la pregunta de facilitar el repaso, la brecha entre estudiantes y docentes es mayor, con una diferencia de 9 puntos porcentuales (Figura 1 estudiantes) $93 \%$ $(n=26)$ en contra $83 \%(n=25)$ (Figura 2 docentes), respectivamente. El estudiantado considera que estas herramientas audiovisuales facilitan más el repaso de la materia (Figura 1). La evaluación más baja se da en el parámetro de atraer la curiosidad: el $72 \%(n=28)$ de estudiantes y $77 \%(n=23)$ de docentes confieren el valor superior de $4 / 4$ al parámetro, que de todas maneras es un rango alto.

En cuanto a las visitas al canal de YouTube, se puede determinar que no solo corresponden a estudiantes y docentes a quienes se les recomendó revisar, por ello se realiza estadística de las visitas desde enero de 2016 a marzo de 2020 (Figura 3).

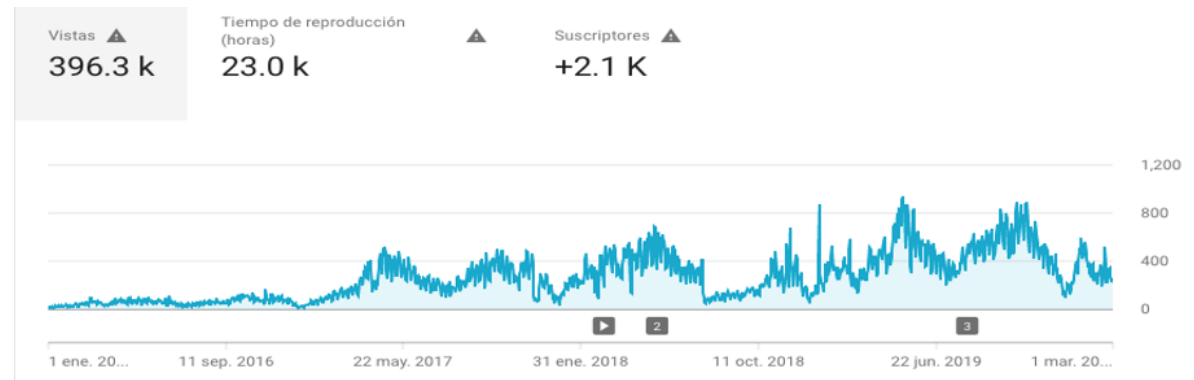

FIGURA 3.

Visitas al canal de YouTube Paulina Moreano, desde el 1 de enero de 2016 al 1 de marzo 2020

Nota: Estadísticas del canal de YouTube Paulina Moreano, visualización del 1 de enero de 2016 al 1 de marzo de 2020. (Moreano, 2020)

Fuente: Moreano, 2020.

En la revisión de las estadísticas en el canal de YouTube, se evidencia que el canal tiene 2100 suscriptores $(+21 \mathrm{~K})$ a marzo de 2020 , con 396.300 visitas y con un promedio de 23 horas de tiempo de reproducción, donde su mayor reproducción fue en el mes de junio del año 2019 (Figura 3). 


\begin{tabular}{|l|l|}
\hline Género del usuario $\downarrow \mathbf{A}$ & + \\
\hline Femenino & $61.4 \%$ \\
\hline Masculino & $38.7 \%$ \\
\hline
\end{tabular}

\begin{tabular}{|c|c|}
\hline Edad del usuario $\downarrow \mathbb{A}$ & Vistas \\
\hline$\square$ Entre 13 y 17 años & $0 \%$ \\
\hline Entre 18 y 24 años & $48.5 \%$ \\
\hline$\square$ Entre 25 y 34 años & $31.9 \%$ \\
\hline Entre 35 y 44 años & $14.1 \%$ \\
\hline$\square$ Entre 45 y 54 años & $4.5 \%$ \\
\hline$\square \quad$ Entre 55 y 64 años & $0.8 \%$ \\
\hline$\square$ A partir de los 65 años & $0.2 \%$ \\
\hline
\end{tabular}

FIGURA 4.

Visitas al canal de YouTube de Paulina Moreano según género y edad de las personas usuarias, desde el 1 de enero de 2016 al 1 de marzo de 2020 Nota: Estadísticas del canal de YouTube Paulina Moreano, visualización del 1 de enero de 2016 al 1 de marzo de 2020 Fuente: Moreano, 2020.

Las visitas al canal con mayor frecuencia corresponden al sexo femenino en un $61.4 \%$. En cuanto al rango de edad con mayores visitas de los usuarios, se establece entre los 18 a 24 años un $48.5 \%$; entre 25 y 34 años un 31.9\%; entre 35 y 44 años un $14.1 \%$; entre 45 a 54 años $4.5 \%$, y otras edades $1 \%$, esto indica un predominio de visitas de adultos jóvenes (Figura 4). 


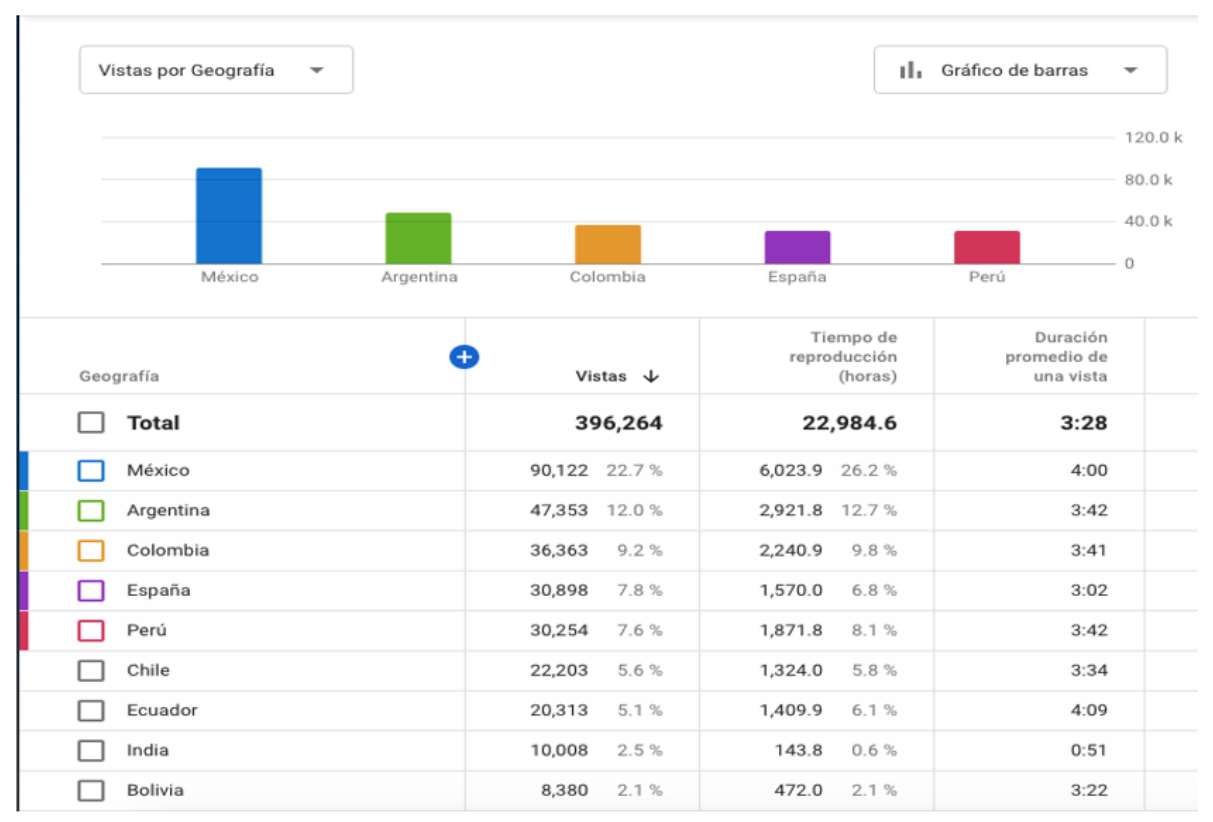

FIGURA 5.

Visitas al canal de YouTube Paulina Moreano según área geográfica del usuario, tiempo de reproducción en horas y duración promedio de visitas, desde el 1 de enero de 2016 al 1 de marzo de 2020

Nota: Estadísticas del canal de YouTube Paulina Moreano, visualización del 1 de enero de 2016 al 1 de marzo de 2020 Fuente: Moreano, 2020.

$\mathrm{Al}$ realizar la revisión de las visitas por área geográfica, la mayor frecuencia corresponde a usuarios con predominio hispano: México con 22.7\%, seguido por Argentina, Colombia, España y Perú. Es importante mencionar que en las estadísticas, aunque con menor frecuencia, aparece la India con el 2,5\% de visitas, sobre el último país de la lista que es Bolivia. Lo anterior determina un nivel de reproducción que excede América Latina (Figura 5).

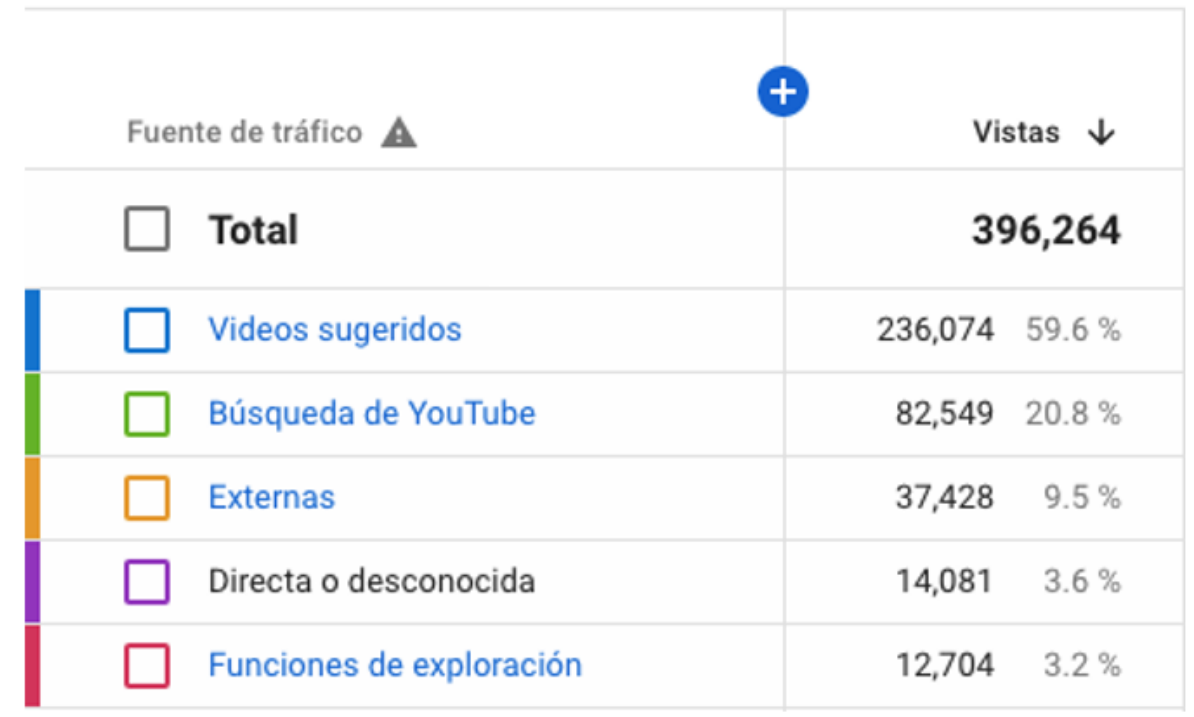

FIGURA 6.

Visitas al canal de YouTube Paulina Moreano según fuente de tráfico, desde el 1 de enero de 2016 al 1 de marzo de 2020

Nota: Estadísticas del Canal de YouTube Paulina Moreano, visualización del 1 ro de enero de 2016 al 1 de marzo de 2020 Fuente: Moreano, 2020. 
En tanto el video con mayor aceptación es el de corazón, con 261885 vistas, seguido por los videos sobre laringe y voz, y audición y equilibrio. La mayor fuente de tráfico es como videos sugeridos, en un 59\%. Las personas usuarias expresan muy buenos comentarios y los videos han sido incrustados en otros medios digitales derivados de la fuente de tráfico prioritaria videos sugeridos. con 59,6\% ( $n=236.074$ visitas), seguido de una fuente autodirigida Búsqueda de YouTube con 20,8\% ( $n=82.548$ visitas) (Figura 6).

\section{Conclusiones y Discusión}

Al analizar los resultados de este estudio, el uso de videos educativos como herramienta de repaso en docencia fue validado como superior $4 / 4$ en un promedio de $85 \%$ del colectivo estudiantil y docente de la PUCE.

La brecha de 9 puntos porcentuales en el indicador facilitación del repaso, entre estudiantes (92\%, Figura 1) y docentes (83\%, Figura 2), puede reflejar el mayor uso de estudiantes de material audiovisual para adquirir conocimiento.

La brecha de 5 puntos porcentuales en el indicador de atraer la curiosidad, (72\%, Figura 1) para estudiantes y (77\%, Figura 2) para docentes, puede reflejar una mayor exposición a material audiovisual como herramienta de aprendizaje por parte de estudiantes y su mayor nivel de exigencia en la elaboración de contenidos novedosos.

Al evaluar el uso de los videos en la red de YouTube se comprueba que se ha logrado llegar a un público más amplio del que se imaginó, que utiliza los videos para fines de repaso y adquisición de conocimiento, y se halla contento de su accesibilidad y contenidos. Se considera que esta es una manera muy eficaz de llegar con docencia de Anatomía y Fisiología al mundo de habla hispana.

El desarrollo de videos educativos, basados en las propias necesidades de una asignatura, cumple con su fin pedagógico, tecnológico y de conocimiento, debido a que se enmarcan desde la misma planificación hasta la evaluación, lo que permite tener un referente de aplicabilidad real.

Los videos educacionales deben estar dentro de un programa instruccional para ser útiles. "El docente debe conseguir la habilidad de hacer reflexionar en la instrucción y de mentalmente estimular acción instruccional" (Hiebert, L., Morris, A., Berk, D., Jansen, A., 2007, p.53). Se requiere poder razonar sobre objetos o situaciones, no solo describirlas, diferenciar información relevante y conectarla, usar diferentes enfoques para reflexionar, evaluar e integrar la información.

Tener en cuenta que existen factores importantes que influyen en aspectos afectivos de la comprensión que son: la narración y la velocidad (Caspi, Gorsky y Privman, 2005).

El diseño, la aplicación y la evaluación de una herramienta educativa predispone la presentación dinámica de la información, así desarrolla un mejor modelo mental (Michas y Berry, 2000). Para la comprensión visual de una herramienta se requiere de una combinación ideal de texto y gráfico o filmación; además, se requiere integrar el contenido narrativo y el educacional para la comprensión visual, lograr que ambos se complementen, que no compitan, para ello, las imágenes requieren ser muy buenas, artísticas, entretenidas que fortalezcan la lectura (Fish, 2000).

El desarrollo de herramientas audiovisuales considera el énfasis en la materia dictada, así como el abordaje en la práctica. Este enfoca su desarrollo en varios niveles del contenido y el proceso de enseñanza, debido a que permiten dividir los procesos en partes pequeñas o cortarlos en fases, todo esto permite recordar información y sirve como un puente entre la teoría y la práctica (Gómez, Sherin, Griesdorn y Finn, 2008; Wisher y Curnow, 1999).

La visualización repetida de procedimientos a través de herramientas audio visuales son muy útiles para el aprendizaje de procedimientos, ya que contienen secuencias de imágenes y audio, e incluso combinación con ocurrencias no descriptibles verbalmente, lo que le permite al estudiantado un aprendizaje significativo (Wanjiang et al., 2014, p.2015). 
La aplicación de los video educativos según Cennamo,1993, citado por Caspi et al., 2005, p.34, pueden percibirse como una herramienta que permite esforzarse menos para aprender; sin embargo, esto dependerá de la calidad y cantidad de la información, además del tiempo que se emplee para la observación de la herramienta educativa.

Las herramientas audiovisuales no solo permiten articular la teoría y la práctica, sino que son importantes y efectivas para estudiantes de bajos resultados, porque ayudan a igualarse con otros, les mejora las notas y la motivación, esto abre el campo de la inclusión educativa, en vista que pueden existir estudiantes con problemas de aprendizaje, que requieren visualizar herramientas más veces y, por lo tanto, se conseguirá el mismo efecto de aprendizaje (Caspi et al., 2005, p.35)

Como limitaciones para la utilización de los videos educativos, y con base en el estudio realizado por Yánez et al. (2016), en el que se evidencia la brecha digital, se puede mencionar:

"Para estudiantes o docentes acostumbrados a estudiar en libros y en clases, puede resultar difícil acostumbrarse a una manera menos rígida y regular de aprendizaje" (Caspi et al. 2005, p.33). El estudiantado está acostumbrado a un sitio fijo, horario establecido y estrategias permanentes de aprendizaje.

Los videos fluyen sin límites claros, no se quedan a lado como un libro, no se subdividen en capítulos, son más difíciles de parar, difíciles de encontrar el punto al retroceder, difíciles de tomar notas. Es importante tener en cuenta que: "algunas estrategias de lectura son inapropiadas para la comprensión visual" (Blomberg, Gamoran, Renkl, Gloger y Seide, 2011).

En su planificación, "la discordancia entre medio y mensaje puede tener resultados contraproducentes en los resultados cognitivos y afectivos" (Caspi et al., 2005, p.33). Algo muy común cuando se usan medios visuales por novelería y no como herramientas de comunicación.

\section{RECOMENDACIONES}

Con base en esta investigación y sus resultados, se recomienda generar mayor cantidad de material audiovisual como herramienta docente. Se comprueba que su utilización es más apreciada por estudiantes que por docentes como una manera adecuada para repasar la materia, pero que a su vez ellos demandan que ese material atraiga más su curiosidad. Por lo tanto, es importante la formación docente actualizada en tecnología, pedagogía y conocimiento de la materia para su producción, sin dejar a un lado el abordaje emocional en su elaboración.

El material audiovisual tipo videos es parte de la realidad tecnológica del mundo actual, están incorporados en gran cantidad de actividades de comunicación. En el área docente deben usarse como herramientas en actividades didácticas, al ser producidos tanto por docentes como estudiantes, utilizados en videoconferencias, en cursos virtuales masivos abiertos, tipo MOOCs, en presentaciones e interacciones en redes sociales.

Otra recomendación de esta investigación es dar acceso al público general al material didáctico audiovisual. Es decir, subirlo a plataformas abiertas para un público que fluctúa entre los 18 a 54 años en un 95\% y en un $61 \%$ de predominio femenino para esta asignatura, ávido por aprender. Además, se recomienda generar más producción en el idioma español, ya que la población de habla hispana sin acceso a información en su idioma es alta. Se evalúa un porcentaje de acceso de los diferentes países de habla hispana, donde México y Argentina suman casi el 40\% de vistas.

Con la tecnología como herramienta, la docencia será cada vez más una actividad de la ciudadanía del mundo para el mundo. 


\section{REFERENCIAS BIBLIOGRÁFICAS}

Blomberg, G., Gamoran, M., Renkl, A., Gloger, I. y Seidel, T. (2011) Understanding Video as a tool for teacher education: investigating instructional strategies to promote reflection. Instructional Science Journal, 42, 443-463, doi: https://doi.org/10.1007/s11251-013-9281-6

Caspi, A., Gorsky, P. y Privman, M. (2005). Viewing Comprehension: Students 'Learning preferences and Strategies when studying from video. Instructional Science Journal, 33, 31-47. Recuperado de https://link.springer.com/a rticle/10.1007\%2Fs11251-004-2576-x

Fierro, C., Fortoul, B. y Rosas, L. (2000). Transformando la práctica docente, una propuesta basada en la investigación acción. México: Paidós.

Fish, S. (2000). A capacity model of children's comprehension of educational content on television. Media Psychology, 2(1), 63-91. doi: https://doi.org/10.1207/S1532785XMEP0201_4

García, B., Loredo, J. y Carranza, G. (2008). Análisis de la práctica educativa de los docentes: pensamiento, interacción y reflexión. Revista Electrónica de Investigación Educativa, 10, 1-15. Recuperado de https://bit.ly/3cnLWM8

Gómez. L., Sherin, M., Griesdorn, J. y Finn, L.E. (2008). Creating social relationships, the role of technology in preservice teacher preparation. Journal of Teacher Education, 59, 117-131. doi: https://doi.org/10.1177/0022 487107314001

Harris, J., Mishra, P. y Koehler, M. (2009). Technological Pedagogical Content Knowledge and Learning Activity Types: Curriculum-based Technology Integration Reframed. Journal of Research on Technology in Education, 41(4), 393-416. doi: https://doi.org/10.1080/15391523.2009.10782536

Hiebert, L., Morris, A., Berk, D., Jansen, A. (2007). Preparing teachers to learn from teaching. Journal of Teaching Education, 58, 47-61. doi: https://doi.org/10.1177/0022487106295726

Michas, I. y Berry, D. (2000). Learning procedural task: effectiveness of multimedia presentations. Applied Cognitive Psychology, 14(6), 555-557. doi: https://onlinelibrary.wiley.com/doi/10.1002/1099-0720(200011/12)14:6\% 3C555::AID-ACP677\%3E3.0.CO;2-4

Mishra, P. y Koehler, M. J. (2006). Technological Pedagogical Content Knowledge: A Framework for Teacher Knowledge. Teachers College Record, 108(6), 1017-1054. doi: https://doi.org/10.1111/j.1467-9620.2006.006 84.x

Morales, M., Trujillo, J. y Raso, F. (2015). Percepciones acerca de la integración del tic en el proceso de enseñanzaaprendizaje de la universidad. Píxel-Bit. Revista de Medios y Educación, 46, 103-117. doi: https://dx.doi.org/1 0.12795/pixelbit.2015.i46.07

Moreano, P. [Paulina Moreano-YouTube]. (2020, marzo 1) Estadísticas del Canal de YouTube [Archivo de video]. Recuperado de https://www.youtube.com/results?search_query=paulina+moreano

Ribayo, M. (2011). Guía para la producción de material Audiovisual. Recuperado de https://bit.ly/3qH3pUR

Valverde, J., Garrido, M. C. y Fernández, R. (2010). Enseñar y aprender con tecnologías: un modelo teórico para las buenas. Educación y cultura en la sociedad de la información, 11(1), 4-445. Recuperado de https://revistas.usal. es/index.php/eks/article/view/5840/5866

Wanjiang, C., Engui, Z., Honghai, W., Zhuping, X., Canming, B., Jian, W. y Lianhong, Li. (2014). Construction and Application of High Quality Medical Video Teaching Resources. En: L. Shaozi, J. Qun Jin, X. Jiang y J. Park (Eds). Frontier and Future Development of Information Technology in Medicine and Education. Lecture Notes in Electrical Engineering. Estados Unidos: Publisher Springer Netherlands.

Wisher, R. y Curnow, C. (1999). Perceptions and effects of Image transmissions during internet-based training. American Journal of Distance Education, 13(3): 37-51, doi: https://doi.org/10.1080/08923649909527034

Yánez, A., Ortiz, L. y Espinosa, V. (2016). Las tecnologías de la comunicación e información (TIC) en salud: un modelo para aplicar en la carrera de Enfermería. Rev. Iberoam. Educ. Investi. Enferm, 6(2), 29-36. Recuperado de: http s://bit.ly/3coekxz 


\section{INFORMACIÓN ADICIONAL}

Cómo citar: Yánez Corrales, A.C. y Moreano Barragan, E.P. (2021). Aplicación de conocimientos docentes en contenido curricular, pedagogía y tecnología a través de herramientas audiovisuales. Revista Educación, 45(2). Recuperado de http://doi.org/10.15517/revedu.v45i1.43469 